\title{
Taming the Master: SWI/SNF chromatin remodeller as a therapeutic target in cancer
}

\author{
Murali Dharan Bashyam ${ }^{1, *}$, Srinivas Animireddy ${ }^{1,2}$ and Pratyusha Bala ${ }^{1,2}$ \\ ${ }^{1}$ Laboratory of Molecular Oncology, Centre for DNA Fingerprinting and Diagnostics, Inner Ring Road, Uppal, Hyderabad 500 039, India \\ ${ }^{2}$ Graduate Studies, Manipal Academy of Higher Education, Manipal 576 104, India
}

\begin{abstract}
Eukaryotic cells use histone modifiers and chromatin remodellers to facilitate protein DNA interactions in the nucleus; an important requisite for regulating several cardinal nuclear processes including transcription, replication, DNA repair and recombination, etc. The SWI/SNF complex is the most well-studied chromatin remodeller and is conserved from yeast to mammals. The complex is recruited to specific DNA sites, where it uses energy from ATP hydrolysis to catalyse nucleosome sliding or histone eviction from DNA. Mutational inactivation of SWI/SNF components has been identified in neurological syndromes and in several cancers. Recent deep sequencing studies have revealed a SWI/SNF mutation frequency of $20 \%$ in cancer genomes. In addition to mutations in tumour samples, extensive studies on cell lines and animal models have revealed tumour suppressive features for many individual SWI/SNF components. Thus, components of the complex are classified as tumour suppressors. Interestingly, however, majority of mutations cause incomplete inactivation of the complex, leaving behind a 'residual' complex that can be targeted for therapy. In addition, characterization of multiple roles of SWI/SNF components has revealed several therapeutic options. The current review summarizes the multi-faceted therapeutic opportunities for tumour bearing mutations in genes, encoding SWI/SNF components.
\end{abstract}

Keywords: ARID1A, chromatin remodeller, SWI/SNF, therapeutic targeting.

\section{Importance of the work}

EFFORTS are ongoing for the past few decades to develop efficient therapeutic options for cancers, where conventional treatments including surgery and chemo/radiation have failed miserably. It is now well-understood that tumours harbour genetic lesions that are essential for their sustenance and therefore provide an attractive target for therapy. An alternative approach is to target specific weaknesses in tumour cells that arise due to mutations in specific genes. This review attempts to discuss several such vulnerabilities that may exist in tumour cells

\footnotetext{
*For correspondence. (e-mail: bashyam69@gmail.com)
}

harbouring mutations in genes encoding components of the SWI/SNF chromatin remodelling complex.

The nucleus of a human cell harbours approximately $2 \mathrm{~m}$ long double-stranded genomic DNA containing 3.3 billion base pairs that are highly compacted to fit inside a 5-10 micron nuclear compartment. To achieve this density, DNA is condensed and tightly packed into a structure called chromatin. Nucleosome, the basic unit of condensed chromatin, includes 146 base pairs of DNA tightly wrapped around a specialized protein structure formed by an octamer of histone proteins. Each histone octamer is composed of two copies each of histones H4, H3, H2A and H2B. The wrapped DNA contacts the histone octamer through various types of noncovalent interactions. The nucleosomal structure provides stability and compactness besides facilitating protection of the genome. However, this arrangement places a huge constraint on proteins that depend on sequence-specific binding to DNA for regulating various nuclear processes such as replication, transcription, DNA repair, recombination, etc. ${ }^{1,2}$. Hence, the chromatin state has to be dynamic, with an inherent ability to switch between a tightly packed and a more 'loosened' state, the latter facilitating binding of proteins to DNA.

Eukaryotic cells employ several mechanisms to covalently modify histones and/or DNA to regulate spatiotemporal accessibility of proteins to DNA. In addition, energy-dependent processes are put in place to facilitate a 'loose' or 'open' chromatin structure, catalysed by chromatin-remodelling enzymes. Histone modifying enzymes catalyse an array of post translational modifications on the C-terminal tail of histones-enabling a change in the active state of chromatin by the recruitment of specific effector proteins. Chromatin remodelling enzymes, on the other hand, catalyse the uncoupling of DNA-histone contacts through nucleosome sliding, eviction, or replacement of canonical histones with histone 'variants' in an ATP dependent manner, thereby exposing underlying DNA sequences (enhancers, promoters, replication origins, sites of DNA recombination or damage) to sequence specific regulators ${ }^{3}$. The ATP-dependent chromatin remodelling enzymes are remarkably diverse and genomewide binding studies have revealed about 20-40,000 sites $^{4}$, indicating the huge abundance of complexes in the cell. A typical chromatin remodeller is a multi-subunit 
Table 1. SWI/SNF proteins from human, drosophila and yeast

\begin{tabular}{|c|c|c|c|c|c|}
\hline \multicolumn{2}{|c|}{ Human } & \multicolumn{2}{|c|}{ Drosophila } & \multicolumn{2}{|c|}{ Yeast } \\
\hline BAF & pBAF & BAP & PBAP & $\mathrm{SWI} / \mathrm{SNF}$ & $\mathrm{RSC}$ \\
\hline BRG1/BRM & BRG1 & \multicolumn{2}{|c|}{ BRM/Brahma } & SWI2/SNF2 & Sth1 \\
\hline \multirow[t]{3}{*}{$\begin{array}{l}\text { ARID1A/ } \\
\text { ARID1B }\end{array}$} & ARID2 & OSA & BAP170 & SWI1/Adr6 & Rsc9 \\
\hline & $\begin{array}{c}\text { BAF180/ } \\
\text { PBRM1 }\end{array}$ & & Polybromo & & Rsc $1,2,4$ \\
\hline & BRD7 & & & & \\
\hline \multicolumn{2}{|c|}{ INI1/BAF47/SNF5 } & \multicolumn{2}{|c|}{ BAP45/SNR1 } & Snf5 & Sfh1 \\
\hline \multicolumn{2}{|c|}{ BAF60a,b,c } & \multicolumn{2}{|c|}{ BAP60 } & Swp73 & Rsc6 \\
\hline \multicolumn{2}{|c|}{ BAF $155 / 170$} & \multicolumn{2}{|c|}{ MMOIRA/BAP155 } & SWI3 & Rsc8/Swh3 \\
\hline \multicolumn{2}{|c|}{ beta Actin } & \multicolumn{2}{|c|}{ Actin } & \multicolumn{2}{|c|}{ Arp 7,9 } \\
\hline \multicolumn{2}{|c|}{ BAF57 } & \multicolumn{2}{|c|}{ BAP111 } & & \\
\hline \multicolumn{2}{|c|}{ BAF53 a,b } & \multicolumn{2}{|c|}{ BAP55/BAP47 } & & \\
\hline \multicolumn{2}{|c|}{ BAF45 a,b,c,d } & & & & \\
\hline
\end{tabular}

assembly consisting of a core ATPase subunit that uses ATP hydrolysis to mobilize nucleosomes. In addition, several (up to 20) non-catalytic protein subunits may be present to provide structural stability to the complex, as well as to impart specificity through interactions with other proteins or complexes. ATP-dependent chromatin remodellers identified till date are categorized into four families based on shared structural or functional domains: (1) SWI/SNF (switch/sucrose non-fermenting), (2) ISWI (imitation-switch), (3) NURD/CHD (chromodomain helicase DNA-binding) and (4) INO80 (inositol-requiring 80). The four complexes function in a non-redundant manner to influence discrete DNA dependent molecular functions. Dysfunction of chromatin remodellers is strongly associated with various developmental defects. More importantly, genetic alterations or aberrant expressions of chromatin remodellers have been identified as possible oncogenic driver in numerous cancer types.

\section{The multi-subunit SWI/SNF chromatin remodelling complex}

The SWI/SNF complex was the first ATP-dependent chromatin remodeller to be identified in the budding yeast Saccharomyces cerevisiae from two independent genetic screens and is the most well characterized till date. The screens revealed genes as transcription co-activators required for regulating mating type switching $(\mathrm{SWI})^{5}$ and sucrose fermentation (sucrose non-fermenting/SNF) ${ }^{6}$. Biochemical purification studies showed that the encoded proteins numbering about 9-12, physically associated to form a relatively large multi-subunit complex that exhibited ATP-dependent nucleosome remodelling activity in vitro $^{7,8}$. Further studies led to identification of additional evolutionarily conserved homologous complexes in S. cerevisiae (the RSC complex) ${ }^{9}$. More importantly, a screen for identifying genes opposing the activation of homeobox genes by the polycomb complex in Drosophila melanogaster yielded similar complexes (BAP and PBAP) ${ }^{10,11}$ and further studies identified counterparts in mammals (the BRG1/BRM - associated factor (BAF) complex ${ }^{12}$ and the polybromo BRG1 - associated factor (pBAF) complex $)^{13}$ (Table 1).

The mammalian SWI/SNF complex is the primary chromatin remodeller for almost all stages of embryonicdevelopment ${ }^{14}$ as well as for differentiation of various cell types during adult life ${ }^{15-17}$. Various proteins that constitute the mammalian SWI/SNF complex can be classified into three categories, namely (a) enzymatic subunits having catalytic ATPase activity including the Brahma homologue (BRM, encoded by SMARCA2) and BRMrelated gene 1 (BRG1, encoded by SMARCA4), (b) set of highly conserved 'core' subunits (SNF5 (also known as, INI1 or BAF47 encoded by SMARCB1), BAF155 and BAF170) and (c) accessory subunits that may contribute towards complex specificity (ARID1A/ARID1B, ARID2, etc.). Recently, a third SWI/SNF complex called noncanonical BAF (ncBAF, also termed GBAF) was identified, that contains GLTSCR1/GLTSCR1L instead of the ARID-domain containing proteins ${ }^{18,19}$.

Only a set of four subunits (BRG1/SMARCA4,SNF5/ SMARCB1, BAF155/SMARCC1 and BAF170/SMARCC2) in humans is necessary and sufficient to perform chromatin remodelling in vitro ${ }^{20}$. However, additional subunits may play a crucial role in binding of complex with chromatin (for example, recognition of acetylated-lysine by components harbouring the Bromo domain) or with transcription factors. Targeting of the complex to specific gene loci is facilitated by the heterogeneity in subunit composition that can manifest differently in different tissues or at different cell states in the same tissue.

\section{Diversity in mammalian SWI/SNF complex composition ensures varied functions}

The size of the BAF complex is estimated to be about $2 \mathrm{MDa}$ making it one of the largest protein complexes in the mammalian cell. The mammalian complex is 
substantially more diverse and multifaceted than the one initially discovered in yeast as it is encoded by at least 29 different genes, suggesting the possibility of assembling a multitude of complexes through combinatorial association. BRG1 or BRM, the two subunits that exhibit ATPase activity, are always present in different SWI/SNF complexes. In addition, the complex comprises three mutually exclusive DNA binding subunits, namely the AT-rich interaction domain 1A (ARID1A/BAF250a), ARID1B (BAF250b) or ARID2. ARID1A/ARID1B constitute disjoint components (paralogs) of the BAF (BRG1/BRM associated factor) complex ${ }^{12}$ whereas ARID2 (BAF180) is a component of the pBAF complex $^{13}$, so named because of the presence of the exclusive PBRM1 subunit ${ }^{11}$. In addition, BRG1 can be present in both BAF and pBAF complexes while BRM is found only in the BAF complex ${ }^{21}$. The recently identified ncBAF complex harbours either GLTSCR1 or GLTSCR1L instead of ARID1A/ARID1B or ARID2 besides either of the two ATPase subunits BRG1 or BRM. The SWI/SNF complex can therefore be broadly classified into three types, namely BAF, pBAF and ncBAF. Several components of the SWI/SNF are encoded by gene families, whose protein products are known to assemble during complex formation in a mutually exclusive fashion despite exhibiting significant similarity in their respective amino acid composition. The general rule for differential complex formation therefore appears to be that, specific position of a subunit in the complex is occupied by any one of the family members (paralogs; position of BAF60 for example, can be occupied by any one of three alternatives BAF60a, b, or c). The mutual exclusivity in SWI/ SNF subunit association is touted as a major mechanism that could in principle give rise to a variety of distinct complexes with differential activities. The alternating subunits cause differential activity as they perhaps project different interacting motifs. Indeed, the subunits of SWI/SNF are known to have various protein-protein and protein-DNA interaction domains including the LXXLL motif, the PHD domain, chromodomains, binding domains that recognize specific DNA structures or ATrich sequences and domains important for recognizing histone marks (like the Bromodomain). The diversity in SWI/SNF subunit composition may mediate the interaction with specific transcription factors and/or modified histones allowing the recognition of specific target gene sets to regulate distinct functions. The ability of BRG1 and BRM for example, to have distinct transcriptional specificity despite an overall amino acid identity of $\sim 70 \%$ is due to $\mathrm{N}$ terminal amino acid sequence variation which allows the interaction with distinct transcription factors ${ }^{22}$. Similarly, ARID1A and ARID1B are mutually exclusive paralogous subunits sharing $60 \%$ amino acid sequence identity but regulate distinct functions ${ }^{23,24}$. The recently identified ncBAF/GBAF complex harbours BRD9, a paralog of the bromodomain containing BRD7, that regu- lates gene sets distinct from that of other BAF and $\mathrm{pBAF}$ complexes $^{18,25}$. Re-configuration of SWI/SNF composition has been shown to be important during developmental processes, especially for lineage specification. For example, the BAF53A and BAF45A subunits are replaced by BAF53B and BAF45B/BAF45C respectively, during differentiation of neural stem cells into mature neurons ${ }^{26}$. Similarly, SWI/SNF containing BAF60a (encoded by SMARCD1) promotes transcription of Oct4 and Sox2 (ref. 4), while the complex harbouring BAF60b (encoded by $S M A R C D 2)$ regulates granulocyte differentiation ${ }^{27}$ and the complex comprising BAF60c (encoded by $S M A R C D 3)$ promotes cardiac and skeletal muscle development $^{28}$.

\section{$\mathrm{SWI} / \mathrm{SNF}$ in disease}

The SWI/SNF complex plays an essential role in epigenetic regulatory mechanisms that impact several developmental processes ${ }^{1}$. Given the fundamental nature of SWI/SNF function, it is but natural to expect the malfunctioning or loss of its subunits to be associated with disease conditions. Of the four distinct mammalian chromatin remodellers (SWI/SNF, ISWI, CHD, INO80), inactivation of SWI/SNF subunits is strongly implicated in various diseases. Indeed, mutations and other forms of genetic lesions in genes encoding SWI/SNF components cause various familial syndromes, multi-system developmental disorders especially related to neuro-development and several types of cancers.

\section{Neuro-developmental disorders}

The SWI/SNF orchestrates various pre- and post-natal neural developmental events besides performing a cardinal role in the proliferation of neuronal stem cells ${ }^{29}$. More importantly, conditioned ablation of BAF155 and BAF170 core subunits causes severe defects in the development of forebrain and related structures ${ }^{30}$, as well as defects in cerebral cortical size and structure ${ }^{31}$ in mice. Based on these observations, alterations and mutations in its components can be expected to cause neuro-developmental defects. Indeed, mutations in several components of the SWI/SNF complex have been reported in intellectual disability syndromes ${ }^{8,9}$. Inactivating mutations in ARID1A, ARID1B, ARID2, SMARCA2, SMARCA4, SMARCB1 and SMARCE1 were reported in Coffin-Siris syndrome $^{10}$. Similarly, missense mutations in SMARCE1, SMARCC1 and SMARCC2 were identified in autism spectrum disorders ${ }^{11,12}$ and mutations in SMARCA2 were detected in patients with Nicolaides-Baraitser syndrome $^{32}$. During the past decade, mutations in several genes encoding SWI/SNF components have been described to be causal to neurological syndromes; the information is summarized in Table 2. 
Table 2. Role of SWI/SNF components in neurological disorders

\begin{tabular}{|c|c|c|c|}
\hline Gene name & Mutation type & Syndrome/symptoms & Reference \\
\hline ARID1A & Nonsense, frameshift, indel & Coffin-Siris syndrome & 127 \\
\hline ARID1B & $\begin{array}{l}\text { Translocation, frameshift, indel, nonsense, } \\
\text { missense, microdeletion }\end{array}$ & $\begin{array}{l}\text { Coffin-Siris syndrome, autism, Nicolaides-Baraitser syndrome, } \\
\text { schizophrenia, Hirschsprung's disease, Intellectual disability }\end{array}$ & $128-131$ \\
\hline ARID2 & Frameshift, indel & Coffin-Siris syndrome & 132 \\
\hline SMARCA2 & $\begin{array}{l}\text { Partial deletion, missense, intronic alteration, } \\
\text { duplication }\end{array}$ & $\begin{array}{l}\text { Coffin-Siris syndrome, Nicolaides-Baraitser syndrome } \\
\text { schizophrenia }\end{array}$ & $133-135$ \\
\hline SMARCB1 & In-frame deletion, missense & $\begin{array}{l}\text { Coffin-Siris syndrome, Kleefstra syndrome phenotypic } \\
\text { spectrum }\end{array}$ & $127,137,138$ \\
\hline SMARCE1 & Missense & Coffin-Siris syndrome & $139-141$ \\
\hline SMARCC1 & Missense & $\begin{array}{l}\text { Autism, social deficits and communication difficulties, } \\
\text { restricted and repetitive behaviours }\end{array}$ & 142 \\
\hline SMARCC2 & Splice site mutation & $\begin{array}{l}\text { Autism, social deficits and communication difficulties, } \\
\text { restricted and repetitive behaviours }\end{array}$ & 142 \\
\hline BCL11A & Micro deletion, missense and frameshift mutations & 2p15-16.1 microdeletion syndrome; autism; schizophrenia & 136,144 \\
\hline ATRX & Missense & ATRX syndrome & 145 \\
\hline SMARCAL1 & $\begin{array}{l}\text { Deletion, missense, nonsense and frameshift } \\
\text { mutations }\end{array}$ & Schimke immuno osseus dysplasia & 146 \\
\hline
\end{tabular}

\section{Human cancers}

More than eleven genes coding various components of the SWI/SNF have been reported to be altered in various cancers (Table 3). The alterations include missense mutations, gene fusions, copy number variations, epigenetic silencing and even overexpression in some cases. The association of SWI/SNF complexes with cancer was first reported in the late 1990s; bi-allelic inactivation of SMARCB1 was identified in the lethal childhood sporadic cancer called Rhabdoid tumours (RT) ${ }^{33-35}$. Germline inactivation of SMARCB1 predisposed patients to $\mathrm{RTs}^{36}$ and a second somatic mutation resulted in full-blown disease. The cardinal role of SMARCB1 in causing RT was confirmed in mice studies; mono-allelic inactivation or conditional ablation causes aggressive rhabdoid-like tumours and T-cell lymphomas ${ }^{17,18}$, whereas a bi-allelic loss resulted in rapid embryonic lethality (as early as 3.5 to 5.5 days post-coitum $)^{16}$. Various types of genetic lesions that inactivate genes encoding various SWI/SNF components including ARID1A, BRG1, ARID1B, BRM, etc. in a wide variety of cancers are described below.

\section{Mutations}

Deep sequencing studies performed over the past decade or so have revealed frequency of mutations in SWI/SNF components in human cancers to be about $20 \%$ (refs 37 and 38). We analysed the mutation frequency of SWI/ SNF in various cancers from the MSK-IMPACT Clinical Sequencing Cohort $^{39}$; the complex was found to be altered in 2150 of $10336(19.6 \%)$ tumour samples, accessed through the cBioPortal (Figure 1), inferring that proper functioning of the polymorphic BAF and $\mathrm{pBAF}$ complexes could be a major mechanism of tumour suppression. The various components of SWI/SNF complex reported to be mutated or altered in human cancers are listed in Table 3. Of the many components, ARIDIA, SMARCA4 and $S M A R C B 1$ exhibit a significantly higher frequency of mutation in cancers. In addition, several subunits exhibit high frequency of mutational inactivation in specific cancer types while harbouring very low or nil mutations in other cancers. For example, SMARCB1 and PBRM1 exhibit a mutation frequency of $95 \%$ and $40 \%$ in Rhabdoid tumours and renal clear cell carcinoma respectively, but are rarely mutated in other cancers ${ }^{40}$. Unlike classical homozygous tumour suppressor events, most SWI/SNF somatic mutational events are mono-allelic indicating a dosage sensitivity or a dominant effect. Only exception is the bi-allelic inactivation of SMARCB1 in RTs, wherein the effect is akin to a classical tumour suppressor.

\section{Epigenetic silencing}

RTs are characterized by a remarkable genetic stability as the tumours exhibit a very low mutation rate; the primary mechanism of carcinogenesis appears to be loss of epigenetic control mediated by the SWI/SNF on the CDKN2A tumour suppressor loci. Thus, RT is perhaps one of the first examples of a cancer arising purely due to a genetic defect that manifests at the epigenetic level. In addition to inactivating mutations, other underlying mechanisms, such as epigenetic silencing through nucleosome remodelling, methylation of histones and DNA, also contribute to the inactivation or loss of function of chromatin remodellers in human cancer. For example, the BRM gene is inactivated in adult lung ${ }^{41}$, breast, ovary, bladder and esophagus cancers, and pediatric rhabdoid tumours 
Table 3. Role of SWI/SNF components in cancers

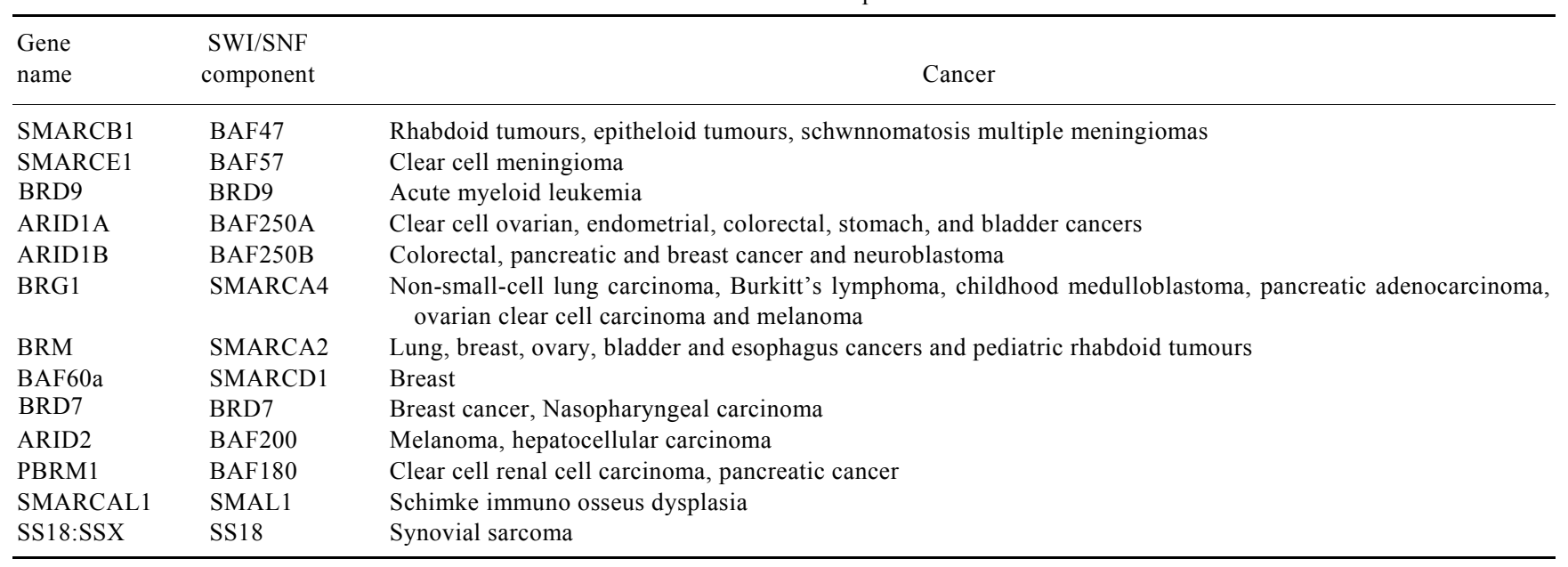

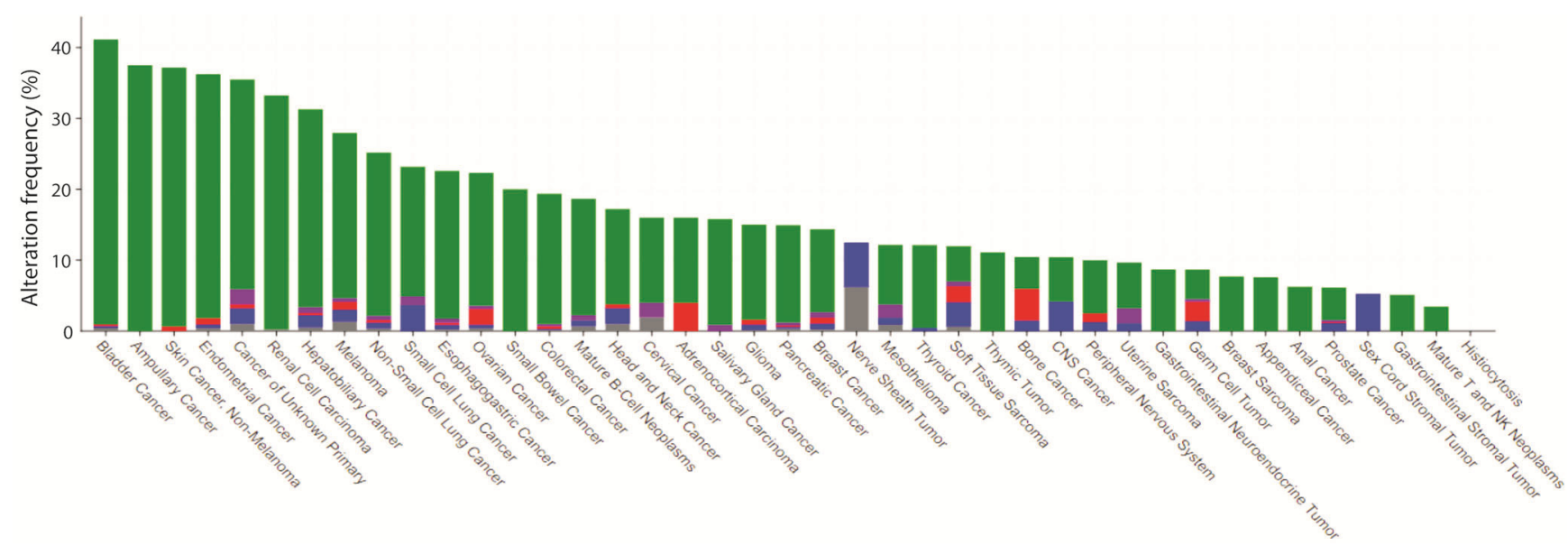

Figure 1. Frequency of SWI/SNF inactivation in various cancers. The mutation frequency was determined from cBioPortal as described in the text. Colour code: green, mutation; red, amplification; blue, deep deletion; purple, fusion; grey, multiple alterations.

through epigenetic silencing by DNA methylation, promoter mutations and other non-mutation modes ${ }^{42}$. In addition, BAF250B exhibits significantly reduced or loss of expression in pancreatic cancer cell lines ${ }^{43}$ because of promoter hyper methylation.

\section{How does SWI/SNF perturbation drive oncogenesis?}

As mentioned above, the SWI/SNF family of chromatin remodellers regulate several cardinal cell processes including transcription, DNA repair and recombination, cell cycle, cell death or survival, differentiation and genomic stability. These could be a direct effect of faulty remodelling of chromatin or an indirect effect of deregulated expression of target genes. Since these processes are expected to be cardinal for tumour suppression and given that SWI/SNF appears to have an extensive footprint across the mammalian genome, it is likely to be involved directly or indirectly in several tumorigenic processes. Earlier studies have elucidated SWI/SNF-directed gene expression programs regulating specific pathways and how mutation in SWI/SNF components promotes cancer. $\mathrm{SWI} / \mathrm{SNF}$ was shown to interact with both $\mathrm{Rb}$ (a tumour suppressor) ${ }^{44}$ and MYC (an oncogene) $)^{24,45}$ leading to perturbations of genes controlled by these master regulators. The complex also binds to and regulates transcriptional program controlled by p53 (ref. 46). In fact, p53 appears to depend on interaction with SWI/SNF components BAF60a/SMARCD1 (ref. 47), SNF5/INI1 (ref. 48), ARID1A/ SMARCF1 (ref. 49) and BRG1/SMARCA4 (ref. 50) to activate its target genes. Thus, SWI/SNF appears to be an important component of the tumour suppressor function performed by $\mathrm{p} 53$. Several reports revealed multi-faceted effect of SWI/SNF on cell cycle progression. Through its interaction with $\mathrm{Rb}, \mathrm{SWI} / \mathrm{SNF}$ regulates transcription of p16 and E2F targets ${ }^{51}$. SWI/SNF induces expression of additional cell cycle regulating and senescence inducing genes such as p21 (refs 43, 52, 53) and p53 (ref. 53) besides being an important mediator of the cell cycle regulation performed by TGF- $\beta$ signaling ${ }^{54,55}$. 
A major role of $\mathrm{SWI} / \mathrm{SNF}$ in regulating tumorigenesis appears to emanate from its antagonistic action on the PRC2/EZH2 (Polycomb group) complex ${ }^{56}$. One of the strongest associations of a SWI/SNF component with tumorigenesis is that of SMARCB1/INI1/SNF5 with RTs. Interestingly, the mechanistic link of SMARCB1 inactivation with tumorigenesis appears to act primarily through antagonism with PRC2/EZH2 mediated histone H3K27 tri methylation ${ }^{57}$. Several additional mechanisms including activation of cyclin D1, perturbation of sonic hedgehog and Wnt signalling have also been proposed $^{57,58}$. Loss of BRG1 promotes tumour aggressiveness in primary and established lung cancer cell lines through perturbations in global chromatin architecture especially in gene loci pertinent for the disease ${ }^{59}$.

Though initial studies focused on evaluating the role of SWI/SNF at or near promoters to facilitate transcription initiation by RNA polymerase II $^{60}$, work during the past few years has provided evidence for their requirement at enhancers especially pertaining to their role in cancers. $\mathrm{SWI} / \mathrm{SNF}$ appears to bind to and recruit $\mathrm{CBP} / \mathrm{p} 300$ histone acetyl transferase at regular (not super) enhancers especially related to genes important for lineage specification $^{61,62}$ and the complex also regulates enhancers that function to establish and maintain cell identity ${ }^{63,64}$. The enhancer regulation property of SWI/SNF appears to play a major role in tumorigenesis as well. Genetic knockout of ARID1A in the mouse colon causes colon cancers through loss of ARID1A containing SWI/SNF activity on enhancers. ARID1B containing complexes, however, maintain activity of a distinct set of enhancers ${ }^{65}$, probably required for tumour sustenance. Similarly, RTs caused by SMARCB1 also emanate from a loss of function of enhancers related to lineage-specific function but not of super-enhancers whose activity is essential for survival of the tumour ${ }^{66}$. Thus, loss of SWI/SNF leads to de-activation of enhancers driving lineage and differentiation-related genes, perhaps resulting in de-differentiation and a proliferative phenotype.

The SWI/SNF pBAF complex is known to play a role in regulating repair of DNA double-strand breaks by promoting transcription repression and this activity is dependent on Ataxia telangiectasia mutated (ATM) ${ }^{67}$. More recently, it was shown that inhibition of $\mathrm{pBAF}$ mediated transcription repression led to increased chromosomal re-arrangements suggesting a possible role in promoting tumorigenesis ${ }^{68}$.

\section{Therapeutic options in tumours harbouring genetic lesions in SWI/SNF components}

About two decades ago, several laboratories began to work towards developing targeted therapies given the poor success achieved with conventional therapies like radiation and chemotherapy. Years of research has yielded targeted therapies with significant success against oncogenic events in specific cancers such as the HER2 amplification in breast cancer (Herceptin/Trastuzumab) ${ }^{69}$, oncogenic gain of function mutation in EGFR (lung cancer) $^{70-72}$, $A L K$ gene rearrangements (also in lung cancer) ${ }^{73}$, and a few others. Despite the initial success, most targeted therapies encountered resistance in a significant proportion of patients and addressing this issue is a major research focus in laboratories the world-over. In addition, efforts were also initiated towards other modes of targeted therapies. One such approach focused on determining vulnerabilities in tumour cells arising out of specific genetic lesions.

\section{Targeting vulnerabilities arising from inactivation of tumour suppressive SWI/SNF}

As already described, inactivating mutations in a SWI/ SNF component appear to occur in about $20 \%$ of all cancers. It was initially thought that genetic lesions in different SWI/SNF components in different cancers had a similar effect on tumorigenesis emanating from inactivation of SWI/SNF function. However, additional observations provided evidence against identical action of various mutations. First, ablation of each component is usually associated with one or a few unique cancer types. For example, loss of ARIDIA is common in ovarian cancer $^{74,75}$, that of SMARCA4 in lung cancer ${ }^{76}$ and of $S M A R C B 1$ in $\mathrm{RTs}^{77}$; this specificity of individual SWI/ SNF subunits has also been validated in mouse models ${ }^{35,78}$. Secondly, mouse knockout studies revealed a distinct phenotype for each SWI/SNF component tested. For example, SMARCB1 and SMARCA4 knockouts were embryonic lethal $^{79,80}$, whereas that of SMARCD3 resulted in abnormal cardiac development ${ }^{81}$.

\section{Inactivation of one SWI/SNF component/complex generates synthetic lethality in another}

Wang and colleagues mooted the idea whether the differential behaviour emanating from inactivation of each component could result from differential activity of the residual complex which in turn could be crucial for tumour cell survival ${ }^{82}$. They tested this hypothesis by ablating SMARCA4 (which encodes the ATPase subunit BRG1) in the background of SMARCB1 loss and the results indeed revealed a dependency of $S M A R C B 1$ ablated cells on SMARCA4. Thus, SMARCA4 loss is synthetic lethal with $S M A R C B 1$ ablation and can be utilized as a therapeutic target. Though the authors proposed that the residual BAF complex could be important for oncogenic potential of the tumour cell, it is possible that this dependency was due to action of the pBAF complex that incorporates BRG1 as the only ATPase subunit; a possibility not considered by the authors. Nevertheless, this observation plus the fact that: (a) previous studies showed 
that the complex could be assembled even in the absence of one component ${ }^{83,84}$ and (b) the SWI/SNF complex is the most important chromatin remodeller during ontogeny and adult life, led to several initiatives directed towards identifying genes that could be synthetic lethal with other inactivated SWI/SNF components. In such a screen, a cancer cell line harbouring inactivation of one SWI/SNF component is used to generate a library of clones each harbouring inactivation of one additional gene; the clones are evaluated for any one of several tumorigenic characteristics (such as survival, growth rate, viability, cell motility/migration, etc.). These screens interestingly revealed a synthetic lethal dependency of ARIDIA mutant cells on $A R I D 1 B$ (loss of one ARID domain BAF subunit makes the cells dependent on the remaining one $)^{85}$ as well as of SMARCA4 inactivated cells on SMARCA2 (loss of one ATPase subunit makes the cells dependent on the remaining one $)^{84,86}$. These results point to the fact that ablation of one SWI/SNF subunit does not de-activate the whole complex as thought earlier, but the remaining complex appears to perform some function crucial for sustenance of the tumour cell. Interestingly, a large-scale proteomic analysis following individual subunit deletion in yeast did reveal presence of active residual SWI/SNF complexes ${ }^{87}$, further supporting the results obtained in humans.

A more recent study revealed cancers with perturbation in BAF components to be dependent on $\mathrm{ncBAF}^{19}$, thus highlighting a synthetic lethal relationship between two distinct SWI/SNF complexes as against paralogs of the same complex. Perhaps perturbation of a core component (for which no paralog is available) makes the cell dependent on a distinct SWI/SNF complex. One common theme that has emerged from these studies, however, is that SWI/SNF complex is essential for tumour maintenance and thus the residual complex offers an excellent therapeutic option. Of course, the therapeutic target in the residual complex will differ depending on the tumour type as well as identification of the inactivated SWI/SNF component.

\section{Vulnerabilities in PRC1/2}

Another approach to identify therapeutic targets in tumours harbouring SWI/SNF inactivation is based on the Polycomb Repressive Complexes (PRC1/PRC2), categorized under Polycomb Group (PcG) of proteins, that exhibit histone methyl transferase enzymatic activity which is antagonistic to the SWI/SNF and can be expected to exhibit augmented oncogenic activation in the background of SWI/SNF inactivation. Initial studies performed in Drosophila provided the first evidence for an antagonistic relationship between the SWI/SNF and PRC2 complexes. During initial stages of Drosophila development, the rapid burst of cell proliferation is primarily driven by the activation of $\mathrm{PcG}$ proteins that includes the PRC1/PRC2 complex. These complexes use their inherent histone methyl transferase activity to transcriptionally silence genes belonging to the Homeobox (HOX) family. During the later stages of development, a reduction in proliferation and initiation of differentiation is triggered by the activation of the Trithorax group of proteins (including members of the SWI/SNF complex) which inhibit PRC2 inducing thereby the expression of HOX genes ${ }^{88}$. The antagonistic relationship between the two complexes has been validated in mammals as well $^{56,89}$. Based on this knowledge, it was hypothesized that RTs that arise due to inactivation of SMARCB1 could be critically dependent on activity of the PRC2 complex; the hypothesis was tested and successfully validated by applying an EZH2 synthetic inhibitor in a mouse xenograft model for malignant rhabdoid tumours ${ }^{90}$. Similar efforts were undertaken for tumours harbouring inactivation of other SWI/SNF components. For example, ARID1A mutated ovarian cancer cells were found to be synthetic lethal for EZH2 inhibition in cell lines and mouse models ${ }^{91,92}$. More importantly, it seems fair to assume that tumours developing due to inactivation of a SWI/SNF component might be dependent on PRC2 proteins for their sustenance. However, whether this can be applicable to a broad range of cancer types is an open question. Of note, there are intrinsic differences in the PRC2-SWI/ SNF antagonism between Drosophila and humans; the Drosophila-BAF complex inhibits action of Polycomb proteins at the HOX locus ${ }^{93}$ whereas in humans, both complexes work synergistically to place the suppressive histone H3K27 trimethylation mark on the HOX loci ${ }^{94}$.

An interesting link between BRG1 and EZH2 was revealed in non-small cell lung cancer. Tumours harbouring genetic inactivation of BRG1 were sensitive to EZH2 inhibition that resulted in increased susceptibility to TopoII inhibitors ${ }^{95}$. However, there is a complete reversal of the response in BRG1 wild type tumours which become resistant to TopoII inhibition upon EZH2 ablation $^{95}$. Therefore, BRG1 status appears to be an important biomarker to predict response to EZH2 and TopoII inhibition in NSCLC.

\section{Vulnerabilities in additional non-SWI/SNF proteins/complexes}

Additional screens revealed a dependency on other nonSWI/SNF genes/complexes. In an siRNA-based screen for essential genes in lung cancer cells exhibiting SMARCA4 loss, Aurora kinase A was identified suggesting importance of SWI/SNF function in centrosome independent mode of mitotic spindle assembly which is probably upregulated in lung cancer ${ }^{96}$. Thus, SWI/SNF functions not directly related to transcriptional regulation could also be evaluated for targeted therapies in tumours exhibiting loss of one SWI/SNF component. 
Screening for a gene which provides a vulnerability target for SWI/SNF mutant cancers is not the only approach employed by researchers for exploring treatment options. In a more standard approach, Deribe et al. ${ }^{97}$ decided to determine transcriptome signature to identify differential pathways in SWI/SNF mutant lung cancer. They performed a comparative transcriptome analysis between KRAS/p53 ablated tumours generated in mice and tumours generated from KRAS/P53/SMARCA4 ablation and identified genes regulating mitochondrial oxidative phosphorylation (OXPHOS) to be enriched in tumours arising out of the triple genetic event compared to KRAS/P53 ablated tumours ${ }^{97}$. These tumours were increasingly dependent on this important mitochondrial function for their energy requirement and vulnerable to small molecular inhibitors of OXPHOS ${ }^{97}$.

The continuing research on SWI/SNF components has revealed several (non-transcriptional) roles which may provide alternative strategies for therapeutics. A study focused on identifying binding partners of ATR, a protein involved in initiation of DNA damage response, revealed ARID1A as a binding partner. Further evaluation revealed essentiality of ARID1A for double-strand break repair as well as for regulating the G2/M DNA damage checkpoint. More importantly, loss of ARID1A makes cells partially deficient for double-strand break repair (similar to the situation arising due to BRCAl mutations) and makes them vulnerable to PARP inhibitors ${ }^{98}$. Given the earlier studies that ascribed an important role for SWI/ SNF in DNA repair, the current discovery of importance of ARID1A in double-strand break repair and synthetic lethality with PARP is not surprising.

Recently, another interesting link between DNA damage repair and SWI/SNF was elucidated. It was shown that SWI/SNF promotes double-strand break and nucleotide excision repair not only by facilitating access of DNA repair proteins by nucleosomal remodelling but also by activating expression of GTF2H1, a component of the TFIIH complex. Interestingly, however, GTF2H1 cannot be used as a synthetic lethal target under ablation of BRG1 or BRM as the cells somehow manage to retain adequate levels of GTF2H1 (ref. 99). Though BRG1 levels were proposed as a marker to predict response to DNA damage-based therapies such as 'Cisplatin', it appears that GTF2H1 levels are a better predictive biomarker. More importantly, it may be important to delineate mechanism of compensation in GTF2H1 levels upon BRG1/BRM ablation which could become a vulnerability that can be exploited for therapy.

\section{Screening for synthetic lethality associated with ARIDIA inactivation}

Since ARID1A is the most frequently mutated SWI/SNF component in ovarian clear cell carcinoma (OCCC), several studies performed genetic screens to identify synthetic lethality with ARID1A mutations. An shRNAbased synthetic lethal 'kinome' screen was performed in a large panel of OCCC cell lines which identified BRD2 as a synthetic lethal target in cell lines having mutant ARID1A which was validated in mouse xenografts derived from OCCC cell lines as well as from patient tumours ${ }^{100}$. Interestingly, using chromatin immunoprecipitation, BRD2 was shown to bind to the promoter regions of several SWI/SNF genes including ARIDIA, ARID1B, SMARCE1/BAF57 and SMARCC2/BAF155, suggesting perhaps that BRD2 might be involved in transcriptional activation of SWI/SNF genes. A separate functional genomics screen using anti-cancer drugs already in use, identified dasatinib as synthetic lethal with ARID1A mutation in OCCCs; dasatinib probably acts by removing the ablation of $\mathrm{p} 21 / \mathrm{Rb} 1$ caused due to ARID1A mutation $^{101}$. A third synthetic lethality screen using shRNAs against eleven histone deacetylases (HDACs) identified HDAC6 to be synthetic lethal with ARID1A mutation $^{102}$. Interestingly, ARID1A appears to be a transcriptional repressor of HDAC6 and mutation induced ARIDIA loss of expression alleviates repression of HDAC6 which in turn acts by inhibiting the apoptosis promoting function of wild type p53 through deacetylation $^{102}$. A separate study based on a proteome screen to detect ARID1A binding partners revealed ARID1A binding to MSH2 (ref. 103). Further, ARID1A was shown to participate in mismatch repair and ARID1A mutations were shown to increase the tumour mutation burden and causing generation of several 'neo-antigens' similar to tumours arising from mismatch repair inactivation resulting in microsatellite instability. More importantly, ARID1A mutant tumours generated in syngeneic mice were sensitive to immune checkpoint therapy ${ }^{103}$. Thus, ARID1A mutations can be used as a biomarker to predict response to immune checkpoint inhibitors.

The repertoire of cell processes where SWI/SNF (particularly the ARID1A subunit) plays a cardinal role seems to be expanding with more and more laboratories reporting new findings. A recent study revealed a unique link between SWI/SNF and the tumour suppressive cytoplasmic Hippo signalling cascade, which represses activity of the YAP/TAZ transcription factors that control cell proliferation in relation to organ size. ARID1A binds to and represses activity of YAP/TAZ. Mechanical stress induced by attainment of optimum organ size and relayed through F-actin dynamics perturbs the interaction between ARID1A and YAP/TAZ allowing functional activation of the latter through binding with TEAD ${ }^{104}$. Thus, loss of SWI/SNF could potentially activate YAP/TAZ function revealing yet another therapeutic vulnerability.

Given the multiple nuclear functions of the SWI/SNF (such as DNA recombination, repair, YAP/TAZ regulation, etc.), one would expect several vulnerabilities arising from its inactivation, some of which are already being evaluated for possible targeted therapies as enumerated 
above. In addition, the possibility of SWI/SNF components exhibiting non-SWI/SNF dependent functions(s) (such as the E3 ubiquitin ligase activity of ARID1B that targets histone $\mathrm{H}_{2} \mathrm{~B}^{105}$ ), provides further opportunities for possible therapeutic targeting. Indeed, we have not yet understood the complete repertoire of SWI/SNF function; work in several laboratories is ongoing in this direction and one can expect additional breakthroughs in the near future.

\section{Direct targeting of oncogenic SWI/SNF?}

Though the SWI/SNF complex is classified as a tumour suppressor, the fact that nucleosome repositioning is likely to be used by several (possibly including oncogenic) cellular processes, it should not be surprising to detect possible oncogenic roles for protein components of the complex. In addition, given the importance of SWI/SNF role in functioning of enhancers, one would expect the complex to perhaps regulate the activation of oncogenes as well. Many studies have reported components of the SWI/SNF to be widely overexpressed in human cancers, such as BRG1 and BRM in acute leukemia ${ }^{106}$, colorectal cancer $^{107}$, prostate cancer ${ }^{108}$ and primary breast can$\operatorname{cers}^{109}$; BAF53a in rhabdomyosarcoma ${ }^{110}$ and glioma ${ }^{111}$; BAF57 in prostate cancer ${ }^{112}$; BAF60a in gastric cancer ${ }^{113}$ and BAF155 in prostate ${ }^{114}$ and colorectal cancer. Leukemia cells appear to rely on BRG1 containing SWI/SNF to maintain their oncogenic status ${ }^{115}$ by activating the $M Y C$ oncogene $e^{106}$. Another study revealed requirement of BRG1 for proliferation of breast cancer cells ${ }^{116,117}$ as well as for the maintenance of neuroblastoma cell viability ${ }^{118}$. The importance of SWI/SNF in activation of oncogenic target genes due to action of the EWSR1-FLI fusion was highlighted recently in Ewing's sarcoma ${ }^{119}$. The EWSR1FLI fusion recruits SWI/SNF to specific enhancers to facilitate expression of oncogenic target genes ${ }^{119}$. A more recent meta-analysis study revealed BRG1 to be routinely overexpressed in several tumour types ${ }^{120}$.

A more direct evidence for oncogenic mode of action of a mutant SWI/SNF was revealed in a study on synovial sarcomas. Synovial sarcoma is defined at the molecular level by a recurrent driver chromosomal translocation $\mathrm{t}(\mathrm{X} ; 18)(\mathrm{p} 11.2 ; \mathrm{q} 11.2)$ resulting in the fusion of SS18, a recently described component of SWI/SNF, with the related protein SSX. Interestingly, the fusion protein incorporates itself into and evicts both SS18 and the core subunit BAF47 from the SWI/SNF complex. The altered complex containing the fusion protein binds to the SOX2 locus relieving the repressive histone H3K27 trimethylation mark thereby inducing $\mathrm{SOX} 2$ expression and cell proliferation. Since the altered complex is recruited to an oncogene (here SOX2), the effect is a propagation of tumorigenic features ${ }^{121,122}$. Interestingly, the action of the altered complex at the $S O X 2$ locus is identical to that of the wild type, i.e. to displace proteins of the polycomb group (here EZH2) and reduce or remove the transcriptionally repressive H3K27 trimethylation mark. These observations provide renewed evidence for the antagonism between EZH2 and SWI/SNF on many gene loci. Thus, the SS18-SSX fusion protein becomes a possible direct target for therapy in synovial sarcomas.

As described above, wild type p53 is known to utilize various SWI/SNF components including SNF5 (ref. 48), BRG1 (ref. 50), ARID1A (ref. 49) and BAF60a (ref. 47) to facilitate its transcriptional activation and tumour suppressor function. TP53 is the most frequently mutated gene in human cancers and specific recurrent mutations (affecting 'hotspot' amino acids located in the region encoding its DNA binding domain) have been shown to exhibit an oncogenic gain of function. Though these mutant p53 forms lose their respective DNA binding activities, they are able to co-operate with other transcription factors to activate expression of oncogenes. Interestingly, several mutant p53 appear to hijack SWI/SNF components to facilitate their oncogenic functions. For example, mutant p53 was shown to cooperate with SWI/SNF to induce the expression of pro-angiogenic factor VEGFR2 in breast cancer cells ${ }^{123}$. Similarly, knockdown of BRG1 was shown to induce senescence in CRC cells through a p21 and p53 dependent mechanism ${ }^{124}$.

Classical tumour suppressor genes (TSGs) and oncogenes harbour distinct mutation pattern in tumours. TSGs often exhibit loss of function (inactivation) mutations such as nonsense or frame shift, whereas mutations in oncogenes cause a gain of function and therefore are mostly of the missense category. While loss-of-function mutations in SWI/SNF subunits seems to be highly prevalent in cancer, missense mutations have also been described $^{125,126}$. It is unclear whether these point mutations result in complete or partial loss of function or potentially an oncogenic gain of function. The preliminary observations enumerated above highlight a possible oncogenic action of SWI/SNF components and could be a prelude to additional discoveries. Future work in this direction may thus reveal whether an oncogenic function of SWI/SNF components could be targeted for therapy in specific tumours.

\section{Concluding remarks}

Decades of research has revealed SWI/SNF to be a master regulator of chromatin dynamics impinging on several cardinal nuclear processes. More importantly, it is one of the most frequently mutated protein complexes in cancer. Despite this, we still know very little about the mechanism of the multi-protein complex in regulating fundamental nuclear processes or tumorigenesis. It is unclear why unlike classical tumour suppressors, SWI/SNF components do not exhibit bi-allelic mutations (with $S M A R C B 1$ 


\section{REVIEW ARTICLES}

being the only exception). It is possible that genes encoding SWI/SNF components are essential and a bi-allelic mutation is lethal (as shown with mice knockout studies) whereas a mono-allelic mutation disrupts the complex due to a dosage effect.

Of note, the susceptibility of a tumour cell harbouring inactivating mutation in a SWI/SNF subunit could emanate from activity of the residual complex or vulnerability of an unrelated function or from the activation of an oncogenic function originally suppressed by SWI/SNF. In contrast, there is also evidence of some cancer types hijacking a possible oncogenic function of SWI/SNF components. It will be important to design therapies based on these contrasting features differing from tumour to tumour and patient to patient, paving the way for therapeutic regimes tailor-made for each cancer type and each patient, what is popularly known as 'precision medicine'. Recent findings have increased our basic knowledge of these mechanisms and revealed unique therapeutic options arising from inactivation of individual SWI/SNF components. Moving forward, furthering our understanding of the basic roles of wild type and mutated SWI/SNF will likely reveal additional therapeutic options for cancer types critically dependent on SWI/SNF function.

1. Narlikar, G. J., Fan, H. Y. and Kingston, R. E., Cooperation between complexes that regulate chromatin structure and transcription. Cell, 2002, 108, 475-487.

2. Wilson, B. G. and Roberts, C. W., Swi/snf nucleosome remodellers and cancer. Nat. Rev. Cancer, 2011, 11, 481-492.

3. Saha, A., Wittmeyer, J. and Cairns, B. R., Chromatin remodelling: the industrial revolution of DNA around histones. Nat. Rev. Mol. Cell Biol., 2006, 7, 437-447.

4. Ho, L., Jothi, R., Ronan, J. L., Cui, K., Zhao, K. and Crabtree, G. R., An embryonic stem cell chromatin remodelling complex, esbaf, is an essential component of the core pluripotency transcriptional network. Proc. Natl. Acad. Sci. USA, 2009, 106 5187-5191.

5. Stern, M., Jensen, R. and Herskowitz, I., Five swi genes are required for expression of the ho gene in yeast. J. Mol. Biol., 1984, 178, 853-868.

6. Neigeborn, L. and Carlson, M., Genes affecting the regulation of suc2 gene expression by glucose repression in saccharomyces cerevisiae. Genetics, 1984, 108, 845-858.

7. Cairns, B. R., Kim, Y. J., Sayre, M. H., Laurent, B. C. and Kornberg, R. D., A multisubunit complex containing the swi1/adr6, swi2/snf2, swi3, snf5, and snf6 gene products isolated from yeast. Proc. Natl. Acad. Sci. USA, 1994, 91, 1950-1954.

8. Cote, J., Quinn, J., Workman, J. L. and Peterson, C. L., Stimulation of gal4 derivative binding to nucleosomal DNA by the yeast swi/snf complex. Science, 1994, 265, 53-60.

9. Cairns, B. R. et al., Rsc, an essential, abundant chromatinremodelling complex. Cell, 1996, 87, 1249-1260.

10. Collins, R. T., Furukawa, T., Tanese, N. and Treisman, J. E., Osa associates with the brahma chromatin remodelling complex and promotes the activation of some target genes. EMBO J., 1999 , 18, 7029-7040.

11. Mohrmann, L., Langenberg, K., Krijgsveld, J., Kal, A. J., Heck, A. J. and Verrijzer, C. P., Differential targeting of two distinct swi/snf-related drosophila chromatin-remodelling complexes. Mol. Cell Biol., 2004, 24, 3077-3088.
12. Wang, W. et al., Purification and biochemical heterogeneity of the mammalian swi-snf complex. EMBO J., 1996, 15, 53705382.

13. Yan, Z. et al., Pbaf chromatin-remodelling complex requires a novel specificity subunit, baf200, to regulate expression of selective interferon-responsive genes. Genes Dev., 2005, 19, 1662-1667.

14. Elfring, L. K. et al., Genetic analysis of brahma: The drosophila homolog of the yeast chromatin remodelling factor swi $/$ snf2. Genetics, 1998, 148, 251-265.

15. de la Serna, I. L., Carlson, K. A. and Imbalzano, A. N., Mammalian swi/snf complexes promote myod-mediated muscle differentiation. Nat. Genet., 2001, 27, 187-190.

16. Gresh, L. et al., The swi/snf chromatin-remodelling complex subunit snf5 is essential for hepatocyte differentiation. EMBO J., 2005, 24, 3313-3324.

17. Pedersen, T. A., Kowenz-Leutz, E., Leutz, A. and Nerlov, C., Cooperation between c/ebpalpha tbp/tfiib and swi/snf recruiting domains is required for adipocyte differentiation. Genes Dev., 2001, 15, 3208-3216.

18. Alpsoy, A. and Dykhuizen, E. C., Glioma tumour suppressor candidate region gene 1 (gltscr1) and its paralog gltscr1-like form swi/snf chromatin remodelling subcomplexes. J. Biol. Chem., 2018, 293, 3892-3903.

19. Michel, B. C. et al., A non-canonical swi/snf complex is a synthetic lethal target in cancers driven by baf complex perturbation. Nat. Cell Biol., 2018, 20, 1410-1420.

20. Phelan, M. L., Sif, S., Narlikar, G. J. and Kingston, R. E., Reconstitution of a core chromatin remodelling complex from swi/snf subunits. Mol. Cell, 1999, 3, 247-253.

21. Hargreaves, D. C. and Crabtree, G. R., Atp-dependent chromatin remodelling: Genetics, genomics and mechanisms. Cell Res., 2011, 21, 396-420.

22. Kadam, S. and Emerson, B. M., Transcriptional specificity of human swi/snf brg1 and brm chromatin remodelling complexes. Mol. Cell, 2003, 11, 377-389.

23. Raab, J. R., Resnick, S. and Magnuson, T., Genome-wide transcriptional regulation mediated by biochemically distinct swi/snf complexes. PLoS Genet., 2015, 11, e1005748.

24. Nagl Jr, N. G., Wang, X., Patsialou, A., Van Scoy, M. and Moran, E., Distinct mammalian swi/snf chromatin remodelling complexes with opposing roles in cell-cycle control. EMBO J., 2007, 26, 752-763.

25. Mashtalir, N. et al., Modular organization and assembly of swi/snf family chromatin remodelling complexes. Cell, 2018, 175, 1272-1288 e1220.

26. Lessard, J. et al., An essential switch in subunit composition of a chromatin remodelling complex during neural development. Neuron, 2007, 55, 201-215.

27. Priam, P., Krasteva, V., Rousseau, P., D'Angelo, G., Gaboury, L., Sauvageau, G. and Lessard, J. A., Smarcd 2 subunit of swi/snf chromatin-remodelling complexes mediates granulopoiesis through a cebpvarepsilon dependent mechanism. Nat. Genet., 2017, 49, 753-764.

28. Puri, P. L. and Mercola, M., Baf60 a, b, and cs of muscle determination and renewal. Genes Dev., 2012, 26, 2673-2683.

29. Taverna, E. G. and Huttner, W. B., The cell biology of neurogenesis: Toward an understanding of the development and evolution of the neocortex. Annu. Rev. Cell Develop. Biol., 2014, 30, 38.

30. Bachmann, C. et al., Mswi/snf (baf) complexes are indispensable for the neurogenesis and development of embryonic olfactory epithelium. PLOS Genet., 2016, 12, e1006274.

31. Tuoc, T. C., Boretius, S., Sansom, S. N., Pitulescu, M. E., Frahm, J., Livesey, F. J. and Stoykova, A., Chromatin regulation by baf170 controls cerebral cortical size and thickness. Dev. Cell, 2013, 25, 256-269. 
32. Van Houdt, J. K. et al., Heterozygous missense mutations in smarca2 cause nicolaides-baraitser syndrome. Nat. Genet., 2012, 44, 445-449, S441.

33. Biegel, J. A., Zhou, J. Y., Rorke, L. B., Stenstrom, C., Wainwright, L. M. and Fogelgren, B., Germ-line and acquired mutations of inil in atypical teratoid and rhabdoid tumours. Cancer Res., 1999, 59, 74-79.

34. Versteege, I. et al., Truncating mutations of hsnf5/inil in aggressive paediatric cancer. Nature, 1998, 394, 203-206.

35. Roberts, C. W., Leroux, M. M., Fleming, M. D. and Orkin, S. H., Highly penetrant, rapid tumourigenesis through conditional inversion of the tumour suppressor gene snf5. Cancer Cell, 2002, 2, 415-425.

36. Eaton, K. W., Tooke, L. S., Wainwright, L. M., Judkins, A. R. and Biegel, J. A., Spectrum of smarcb1/inil mutations in familial and sporadic rhabdoid tumours. Pediatr. Blood Cancer, 2011, 56, $7-15$.

37. Kadoch, C. and Crabtree, G. R., Mammalian swi/snf chromatin remodelling complexes and cancer: Mechanistic insights gained from human genomics. Sci. Adv., 2015, 1, e1500447.

38. Shain, A. H. and Pollack, J. R., The spectrum of swi/snf mutations, ubiquitous in human cancers. PLoS ONE, 2013, 8, e55119.

39. Zehir, A. et al., Mutational landscape of metastatic cancer revealed from prospective clinical sequencing of 10,000 patients. Nat. Med., 2017, 23, 703-713.

40. Masliah-Planchon, J. B., Gindbretiere, J.-M., Bourdeaut, F. and Delattre, O., Swi/snf chromatin remodelling and human malignacies. Annu. Rev. Pathol., 2015, 10, 27.

41. Wu, J., He, K., Zhang, Y., Song, J., Shi, Z., Chen, W. and Shao, $Y$., Inactivation of smarca2 by promoter hypermethylation drives lung cancer development. Gene, 2018, 687, 193-199.

42. Marquez, S. B., Thompson, K. W., Lu, L. and Reisman, D., Beyond mutations: additional mechanisms and implications of swi/snf complex inactivation. Front Oncol., 2014, 4, 372.

43. Khursheed, M. et al., Arid1b, a member of the human swi/snf chromatin remodelling complex, exhibits tumour-suppressor activities in pancreatic cancer cell lines. Br. J. Cancer, 2013, 108, 2056-2062.

44. Trouche, D., Le Chalony, C., Muchardt, C., Yaniv, M. and Kouzarides, T., Rb and hbrm cooperate to repress the activation functions of e2f1. Proc. Natl. Acad. Sci. USA, 1997, 94, 11268 11273 .

45. Cheng, S. W., Davies, K. P., Yung, E., Beltran, R. J., Yu, J. and Kalpana, G. V., C-myc interacts with ini1/hsnf5 and requires the swi/snf complex for transactivation function. Nat. Genet., 1999, 22, 102-105.

46. Lee, D., Kim, J. W., Seo, T., Hwang, S. G., Choi, E. J. and Choe, $\mathrm{J}$., Swi/snf complex interacts with tumour suppressor p53 and is necessary for the activation of p53-mediated transcription. J. Biol. Chem., 2002, 277, 22330-22337.

47. Oh, J., Sohn, D. H., Ko, M., Chung, H., Jeon, S. H. and Seong, R. H., Baf60a interacts with p53 to recruit the swi/snf complex. J. Biol. Chem., 2008, 283, 11924-11934.

48. Xu, Y., Yan, W. and Chen, X., Snf5, a core component of the swi/snf complex, is necessary for p53 expression and cell survival, in part through eif4e. Oncogene, 2010, 29, 4090-4100.

49. Guan, B., Wang, T. L. and Shih Ie, M., Aridla, a factor that promotes formation of swi/snf-mediated chromatin remodelling, is a tumour suppressor in gynecologic cancers. Cancer Res., 2011, 71, 6718-6727.

50. Naidu, S. R., Love, I. M., Imbalzano, A. N., Grossman, S. R. and Androphy, E. J., The swi/snf chromatin remodelling subunit brg1 is a critical regulator of p53 necessary for proliferation of malignant cells. Oncogene, 2009, 28, 2492-2501.

51. Betz, B. L., Strobeck, M. W., Reisman, D. N., Knudsen, E. S. and Weissman, B. E., Re-expression of hsnf5/ini1/baf47 in pediatric tumour cells leads to g1 arrest associated with induction of p16ink4a and activation of rb. Oncogene, 2002, 21, 51935203.

52. Inoue, H., Giannakopoulos, S., Parkhurst, C. N., Matsumura, T., Kono, E. A., Furukawa, T. and Tanese, N., Target genes of the largest human swi/snf complex subunit control cell growth. Biochem. J., 2011, 434, 83-92.

53. Tordella, L. et al., Swi/snf regulates a transcriptional program that induces senescence to prevent liver cancer. Genes Dev., 2016, 30, 2187-2198.

54. Xi, Q., He, W., Zhang, X. H., Le, H. V. and Massague, J., Genome-wide impact of the brg1 swi/snf chromatin remodeller on the transforming growth factor beta transcriptional program. J. Biol. Chem., 2008, 283, 1146-1155.

55. Ross, S., Cheung, E., Petrakis, T. G., Howell, M., Kraus, W. L. and Hill, C. S., Smads orchestrate specific histone modifications and chromatin remodelling to activate transcription. EMBO J., 2006, 25, 4490-4502.

56. Wilson, B. G. et al., Epigenetic antagonism between polycomb and swi/snf complexes during oncogenic transformation. Cancer Cell, 2010, 18, 316-328.

57. Kim, K. H. and Roberts, C. W., Mechanisms by which smarcb1 loss drives rhabdoid tumour growth. Cancer Genet., 2014, 207, 365-372.

58. Kohashi, K. and Oda, Y., Oncogenic roles of smarcb1/ini1 and its deficient tumours. Cancer Sci., 2017, 108, 547-552.

59. Orvis, T. et al., Brg1/smarca4 inactivation promotes non-small cell lung cancer aggressiveness by altering chromatin organization. Cancer Res., 2014, 74, 6486-6498.

60. Vorobyeva, N. E., Soshnikova, N. V., Nikolenko, J. V., Kuzmina, J. L., Nabirochkina, E. N., Georgieva, S. G. and Shidlovskii, Y. V., Transcription coactivator sayp combines chromatin remodeller brahma and transcription initiation factor tfiid into a single supercomplex. Proc. Natl. Acad. Sci. USA, 2009, 106, 11049-11054.

61. Alver, B. H. et al., The swi/snf chromatin remodelling complex is required for maintenance of lineage specific enhancers. Nat. Commun., 2017, 8, 14648.

62. Alexander, J. M., Hota, S. K., He, D., Thomas, S., Ho, L., Pennacchio, L. A. and Bruneau, B. G., Brg1 modulates enhancer activation in mesoderm lineage commitment. Development, 2015, 142, 1418-1430.

63. Bossen, C., Murre, C. S., Chang, A. N., Mansson, R., Rodewald, H. R. and Murre, C., The chromatin remodeller brg1 activates enhancer repertoires to establish b cell identity and modulate cell growth. Nat. Immunol., 2015, 16, 775-784.

64. Yu, Y. et al., Olig2 targets chromatin remodellers to enhancers to initiate oligodendrocyte differentiation. Cell, 2013, 152, 248-261.

65. Mathur, R. et al., Arid1a loss impairs enhancer-mediated gene regulation and drives colon cancer in mice. Nat. Genet., 2017, 49, 296-302.

66. Wang, X. et al., Smarcb1-mediated swi/snf complex function is essential for enhancer regulation. Nat. Genet., 2017, 49, 289295.

67. Shanbhag, N. M., Rafalska-Metcalf, I. U., Balane-Bolivar, C., Janicki, S. M. and Greenberg, R. A., Atm-dependent chromatin changes silence transcription in cis to DNA double-strand breaks. Cell, 2010, 141, 970-981.

68. Meisenberg, C. et al., Repression of transcription at DNA breaks requires cohesin throughout interphase and prevents genome instability. Mol. Cell, 2019, 73, 212-223 e217.

69. Tripathy, D. et al., Safety of treatment of metastatic breast cancer with trastuzumab beyond disease progression. J. Clin. Oncol., 2004, 22, 1063-1070.

70. Lynch, T. J. et al., Activating mutations in the epidermal growth factor receptor underlying responsiveness of non-small-cell lung cancer to gefitinib. N. Engl. J. Med., 2004, 350, 2129-2139. 
71. Paez, J. G. et al., Egfr mutations in lung cancer: correlation with clinical response to gefitinib therapy. Science, 2004, 304, 14971500.

72. Pao, W. et al., Egf receptor gene mutations are common in lung cancers from 'never smokers' and are associated with sensitivity of tumours to gefitinib and erlotinib. Proc. Natl. Acad. Sci. USA, 2004, 101, 13306-13311.

73. Shaw, A. T. and Engelman, J. A., Alk in lung cancer: past, present and future. J. Clin. Oncol., 2013, 31, 1105-1111.

74. Wiegand, K. C. et al., Aridla mutations in endometriosisassociated ovarian carcinomas. N. Engl. J. Med., 2010, 363, 1532-1543.

75. Jones, S. et al., Frequent mutations of chromatin remodelling gene arid1a in ovarian clear cell carcinoma. Science, 2010, 330 228-231.

76. Rodriguez-Nieto, S. et al., Massive parallel DNA pyrosequencing analysis of the tumour suppressor brg1/smarca4 in lung primary tumours. Hum. Mutat., 2011, 32, E1999-E2017.

77. Lee, R. S. et al., A remarkably simple genome underlies highly malignant pediatric rhabdoid cancers. J. Clin. Invest., 2012, 122 2983-2988.

78. Bultman, S. J., Herschkowitz, J. I., Godfrey, V., Gebuhr, T. C., Yaniv, M., Perou, C. M. and Magnuson, T., Characterization of mammary tumours from brg1 heterozygous mice. Oncogene, 2008, 27, 460-468.

79. Guidi, C. J. et al., Disruption of ini1 leads to peri-implantation lethality and tumourigenesis in mice. Mol. Cell Biol., 2001, 21 , 3598-3603.

80. Klochendler-Yeivin, A., Fiette, L., Barra, J., Muchardt, C., Babinet, C. and Yaniv, M., The murine snf5/ini1 chromatin remodelling factor is essential for embryonic development and tumour suppression. EMBO Rep., 2000, 1, 500-506.

81. Lickert, H. et al., Baf60c is essential for function of baf chromatin remodelling complexes in heart development. Nature, 2004, 432, 107-112.

82. Wang, X., Sansam, C. G., Thom, C. S., Metzger, D., Evans, J. A., Nguyen, P. T. and Roberts, C. W., Oncogenesis caused by loss of the snf5 tumour suppressor is dependent on activity of brg1, the atpase of the swi/snf chromatin remodelling complex. Cancer Res., 2009, 69, 8094-8101.

83. Doan, D. N., Veal, T. M., Yan, Z., Wang, W., Jones, S. N. and Imbalzano, A. N., Loss of the inil tumour suppressor does not impair the expression of multiple brg1-dependent genes or the assembly of swi/snf enzymes. Oncogene, 2004, 23, 3462-3473.

84. Hoffman, G. R. et al., Functional epigenetics approach identifies $\mathrm{brm} / \mathrm{smarca} 2$ as a critical synthetic lethal target in brg1-deficien cancers. Proc. Natl. Acad. Sci. USA, 2014, 111, 3128-3133.

85. Helming, K. C. et al., Arid1b is a specific vulnerability in arid1amutant cancers. Nat. Med., 2014, 20, 251-254.

86. Wilson, B. G. et al., Residual complexes containing smarca2 (brm) underlie the oncogenic drive of smarca4 (brg1) mutation. Mol. Cell Biol., 2014, 34, 1136-1144.

87. Dutta, A. et al., Composition and function of mutant swi/snf complexes. Cell Rep., 2017, 18, 2124-2134.

88. Schuettengruber, B., Chourrout, D., Vervoort, M., Leblanc, B and Cavalli, G., Genome regulation by polycomb and trithorax proteins. Cell, 2007, 128, 735-745.

89. Kia, S. K., Gorski, M. M., Giannakopoulos, S. and Verrijzer, C. P., Swi/snf mediates polycomb eviction and epigenetic reprogramming of the ink4b-arf-ink4a locus. Mol. Cell Biol., 2008, 28, 3457-3464.

90. Knutson, S. K. et al., Durable tumour regression in genetically altered malignant rhabdoid tumours by inhibition of methyltransferase ezh2. Proc. Natl. Acad. Sci. USA, 2013, 110, 7922-7927.

91. Bitler, B. G. et al., Synthetic lethality by targeting ezh2 methyltransferase activity in arid1a-mutated cancers. Nat. Med., 2015, 21, 231-238.
92. Bitler, B. G., Fatkhutdinov, N. and Zhang, R., Potential therapeutic targets in arid1a-mutated cancers. Expert Opin. Ther. Targets, 2015, 19, 1419-1422.

93. Tamkun, J. W., Deuring, R., Scott, M. P., Kissinger, M., Pattatucci, A. M., Kaufman, T. C. and Kennison, J. A., Brahma: a regulator of drosophila homeotic genes structurally related to the yeast transcriptional activator snf2/swi2. Cell, 1992, 68, 561572.

94. Ho, L., Miller, E. L., Ronan, J. L., Ho, W. Q., Jothi, R. and Crabtree, G. R., Esbaf facilitates pluripotency by conditioning the genome for lif/stat3 signalling and by regulating polycomb function. Nat. Cell Biol., 2011, 13, 903-913.

95. Fillmore, C. M. et al., Ezh2 inhibition sensitizes brg1 and egfr mutant lung tumours to topoii inhibitors. Nature, 2015, 520, 239-242.

96. Tagal, V. et al., Smarca4-inactivating mutations increase sensitivity to aurora kinase a inhibitor vx-680 in non-small cell lung cancers. Nat. Commun., 2017, 8, 14098.

97. Deribe, Y. L. et al., Author correction: Mutations in the swi/snf complex induce a targetable dependence on oxidative phosphorylation in lung cancer. Nat. Med., 2018, 24, 1627.

98. Shen, J. et al., Arid1a deficiency impairs the DNA damage checkpoint and sensitizes cells to parp inhibitors. Cancer Discov., 2015, 5, 752-767.

99. Ribeiro-Silva, C. et al., DNA damage sensitivity of swi/snfdeficient cells depends on tfiih subunit p62/gtf2h1. Nat. Commun., 2018, 9, 4067.

100. Berns, K. et al., Arid1a mutation sensitizes most ovarian clear cell carcinomas to bet inhibitors. Oncogene, 2018, 37, 46114625 .

101. Miller, R. E. et al., Synthetic lethal targeting of arid1a-mutant ovarian clear cell tumours with dasatinib. Mol. Cancer Ther., 2016, 15, 1472-1484.

102. Bitler, B. G. et al., Arid1a-mutated ovarian cancers depend on hdac6 activity. Nat. Cell Biol., 2017, 19, 962-973.

103. Shen, J. et al., Aridla deficiency promotes mutability and potentiates therapeutic antitumour immunity unleashed by immune checkpoint blockade. Nat. Med., 2018, 24, 556-562.

104. Chang, L. et al., The swi/snf complex is a mechanoregulated inhibitor of yap and taz. Nature, 2018, 563, 265-269.

105. Li, X. S., Trojer, P., Matsumura, T., Treisman, J. E. and Tanese, N., Mammalian swi/snf - a subunit baf250/arid1 is an e3 ubiquitin ligase that targets histone h2b. Mol. Cell Biol., 2010, 30, 1673-1688

106. Shi, J. et al., Role of swi/snf in acute leukemia maintenance and enhancer-mediated myc regulation. Genes Dev., 2013, 27, 26482662.

107. Watanabe, T., Semba, S. and Yokozaki, H., Regulation of pten expression by the swi/snf chromatin-remodelling protein brg1 in human colorectal carcinoma cells. Br. J. Cancer, 2011, 104, 146154.

108. Sun, A., Tawfik, O., Gayed, B., Thrasher, J. B., Hoestje, S., Li, C. and Li, B., Aberrant expression of swi/snf catalytic subunits brg1/brm is associated with tumour development and increased invasiveness in prostate cancers. Prostate, 2007, 67, 203-213.

109. Do, S. I. et al., Increased brahma-related gene 1 expression predicts distant metastasis and shorter survival in patients with invasive ductal carcinoma of the breast. Anticancer Res., 2016 36, 4873-4882.

110. Taulli, R. et al., Failure to downregulate the baf53a subunit of the swi/snf chromatin remodelling complex contributes to the differentiation block in rhabdomyosarcoma. Oncogene, 2014, 33, 2354-2362.

111. Meng, L., Wang, X., Liao, W., Liu, J., Liao, Y. and He, Q., Baf53a is a potential prognostic biomarker and promotes invasion and epithelial-mesenchymal transition of glioma cells. Oncol. Rep., 2017, 38, 3327-3334. 
112. Balasubramaniam, S. et al., Aberrant baf57 signaling facilitates prometastatic phenotypes. Clin. Cancer Res., 2013, 19, $2657-$ 2667.

113. Shen, J. et al., Epigenetic silencing of mir-490-3p reactivates the chromatin remodeller smarcdl to promote helicobacter pyloriinduced gastric carcinogenesis. Cancer Res., 2015, 75, 754-765.

114. Heeboll, S. et al., Smarcc1 expression is upregulated in prostate cancer and positively correlated with tumour recurrence and dedifferentiation. Histol. Histopathol., 2008, 23, 1069-1076.

115. Buscarlet, M. et al., Essential role of brg, the atpase subunit of baf chromatin remodelling complexes, in leukemia maintenance. Blood, 2014, 123, 1720-1728.

116. $\mathrm{Wu}, \mathrm{Q}$. et al., The swi/snf atpases are required for triple negative breast cancer cell proliferation. J. Cell Physiol., 2015, 230, 2683-2694.

117. Wu, Q., Lian, J. B., Stein, J. L., Stein, G. S., Nickerson, J. A. and Imbalzano, A. N., The brg1 atpase of human swi/snf chromatin remodelling enzymes as a driver of cancer. Epigenomics, 2017, 9, 919-931.

118. Jubierre, L. et al., Brg1/smarca4 is essential for neuroblastoma cell viability through modulation of cell death and survival pathways. Oncogene, 2016, 35, 5179-5190.

119. Boulay, G. et al., Cancer-specific retargeting of baf complexes by a prion-like domain. Cell, 2017, 171, 163-178 e119.

120. Guerrero-Martinez, J. A. and Reyes, J. C., High expression of smarca4 or smarca2 is frequently associated with an opposite prognosis in cancer. Sci. Rep., 2018, 8, 2043.

121. Kadoch, C. and Crabtree, G. R., Reversible disruption of mswi/snf (baf) complexes by the ss18-ssx oncogenic fusion in synovial sarcoma. Cell, 2013, 153, 71-85.

122. McBride, M. J. et al., The ss18-ssx fusion oncoprotein hijacks baf complex targeting and function to drive synovial sarcoma. Cancer Cell, 2018, 33, 1128-1141 e1127.

123. Pfister, N. T. et al., Mutant p53 cooperates with the swi/snf chromatin remodelling complex to regulate vegfr2 in breast cancer cells. Genes Dev., 2015, 29, 1298-1315.

124. Wang, G. et al., Loss of brg1 induces crc cell senescence by regulating p53/p21 pathway. Cell Death Dis., 2017, 8, e2607.

125. Robinson, G. et al., Novel mutations target distinct subgroups of medulloblastoma. Nature, 2012, 488, 43-48.

126. Pugh, T. J. et al., Medulloblastoma exome sequencing uncovers subtype-specific somatic mutations. Nature, 2012, 488, 106-110.

127. Tsurusaki, Y. et al., Mutations affecting components of the swi/snf complex cause coffin-siris syndrome. Nat. Genet., 2012 , 44, 376-378.

128. Backx, L., Seuntjens, E., Devriendt, K., Vermeesch, J. and Van Esch, H., A balanced translocation $\mathrm{t}(6 ; 14)(\mathrm{q} 25.3 ; \mathrm{q} 13.2)$ leading to reciprocal fusion transcripts in a patient with intellectual disability and agenesis of corpus callosum. Cytogenet Genome Res., 2011, 132, 135-143.

129. Halgren, C. et al., Corpus callosum abnormalities, intellectual disability, speech impairment, and autism in patients with haploinsufficiency of arid1b. Clin Genet., 2012, 82, 248-255.

130. Takenouchi, T. et al., Hirschsprung disease as a yet undescribed phenotype in a patient with arid1b mutation. Am. J. Med. Genet A, 2016, 170, 3249-3252.

131. Santen, G. W. et al., Mutations in swi/snf chromatin remodelling complex gene arid1b cause coffin-siris syndrome. Nat. Genet. 2012, 44, 379-380.
132. Bramswig, N. C. et al., Heterozygosity for arid2 loss-of-function mutations in individuals with a coffin-siris syndrome-like phenotype. Hum. Genet., 2017, 136, 297-305.

133. Koga, M. et al., Involvement of smarca $2 /$ brm in the swi/snf chromatin-remodelling complex in schizophrenia. Hum. Mol. Genet., 2009, 18, 2483-2494.

134. Tang, S., Hughes, E., Lascelles, K., Euro, E. R. E. S. M. A. E. W. G., Simpson, M. A. and Pal, D. K., New smarca2 mutation in a patient with nicolaides-baraitser syndrome and myoclonic astatic epilepsy. Am. J. Med. Genet A, 2017, 173, 195-199.

135. Wolff, D. et al., In-frame deletion and missense mutations of the c-terminal helicase domain of smarca2 in three patients with nicolaides-baraitser syndrome. Mol. Syndromol., 2012, 2, 237-244.

136. De Rubeis, S. et al., Synaptic, transcriptional and chromatin genes disrupted in autism. Nature, 2014, 515, 209-215.

137. Kleefstra, T. et al., Disruption of an ehmt1-associated chromatinmodification module causes intellectual disability. Am. J. Hum. Genet., 2012, 91, 73-82.

138. Gossai, N., Biegel, J. A., Messiaen, L., Berry, S. A. and Moertel, C. L., Report of a patient with a constitutional missense mutation in smarcb1, coffin-siris phenotype, and schwannomatosis. Am. J. Med. Genet. A, 2015, 167, 3186-3191.

139. Wieczorek, D. et al., A comprehensive molecular study on coffin-siris and nicolaides-baraitser syndromes identifies a broad molecular and clinical spectrum converging on altered chromatin remodelling. Hum. Mol. Genet., 2013, 22, 5121-5135.

140. Kosho, T., Okamoto, N. and Coffin-Siris Syndrome International, C., Genotype-phenotype correlation of coffin-siris syndrome caused by mutations in smarcb1, smarca4, smarce1, and arid1a. Am. J. Med. Genet C Semin Med. Genet., 2014, 166, 262-275.

141. Zarate, Y. A. et al., Smarce1, a rare cause of coffin-siris syndrome: clinical description of three additional cases. Am. J. Med. Genet A, 2016, 170, 1967-1973.

142. Neale, B. M. et al., Patterns and rates of exonic de novo mutations in autism spectrum disorders. Nature, 2012, 485, 242245.

143. O'Roak, B. J. et al., Sporadic autism exomes reveal a highly interconnected protein network of de novo mutations. Nature, 2012, 485, 246-250.

144. Bagheri, H. et al., Identifying candidate genes for $2 \mathrm{p} 15 \mathrm{p} 16.1$ microdeletion syndrome using clinical, genomic, and functional analysis. JCI Insight, 2016, 1, e85461.

145. Ratnakumar, K. and Bernstein, E., Atrx: the case of a peculiar chromatin remodeller. Epigenetics, 2013, 8, 3-9.

146. Elizondo, L. I. et al., Schimke immuno-osseous dysplasia: Smarcal1 loss-of-function and phenotypic correlation. J. Med. Genet., 2009, 46, 49-59.

ACKNOWLEDGEMENTS. The work was supported by a grant (BT/PR13948/BRB/10/1406/2015) from the Department of Biotechnology, Government of India to MDB. SA and PB, registered Ph D students of Manipal Academy of Higher Education, Manipal, Karnataka, India, are grateful to the Department of Science and Technology, Government of India, and the University Grants Commission, Government of India respectively, for junior and senior research fellowships.

Received 3 January 2019; revised accepted 7 February 2019

doi: $10.18520 / \mathrm{cs} / \mathrm{v} 116 / \mathrm{i} 10 / 1653-1665$ 